\title{
Modeling of the TeV cosmic-ray anisotropy based on intensity mapping in an MHD-simulated heliosphere
}

\section{T. K. Sako* on behalf of the Tibet AS $\gamma$ Collaboration, and N. V. Pogorelov ${ }^{\dagger}$}

(a complete list of authors can be found at the end of the proceedings)

Small but significant anisotropic features with amplitudes of $\sim 0.1 \%$ have been reported in the arrival directions of galactic cosmic rays at $\mathrm{TeV}$ energies. In this presentation, we preform the modeling of the $\mathrm{TeV}$ cosmic-ray anisotropy outside the heliosphere using experimental data of the Tibet AS $\gamma$ experiment based on the idea of Liouville mapping. In the intensity-mapping process, we take into account for the first time the rigidity distribution of cosmic-ray particles observed by the experiment. We also improve the modeling of the cosmic-ray intensity distribution at the outer boundary outside the heliosphere to improve the reduced $\chi^{2}$ of the fitting. Small structures with angular scales of $\sim 10^{\circ}$ are indicated in the intensity distribution at the outer boundary.

$37^{\text {th }}$ International Cosmic Ray Conference (ICRC 2021)

July 12 th - 23rd, 2021

Online - Berlin, Germany

\footnotetext{
${ }^{\dagger}$ Department of Space Science and Center for Space Plasma and Aeronomic Research, University of Alabama in Huntsville, 320 Sparkman Drive, Huntsville, AL 35899, USA

${ }^{*}$ Presenter
} 


\section{Introduction}

Arrival directions of galactic cosmic rays observed at the Earth are not completely uniform. At $\mathrm{TeV}$ energies some recent high-statistics experiments (e.g. [1-6]) have reported small yet significant anisotropic features with amplitudes of roughly $0.1 \%$, such as a large-scale deficit region called 'Loss-Cone' and an excess region called 'Tail-In'. The origins of these structures have not been known yet, although the anisotropy is considered to reflect how cosmic rays propagate through magnetic fields in the heliosphere and the surrounding interstellar medium.

Some recent studies [7, 8] make use of the 'intensity-mapping' method, in which heliospheric magnetic field structures are reconstructed by MHD simulations, trajectories of cosmic rays are calculated in the MHD model heliosphere, and the cosmic-ray intensity distribution observed at the Earth is mapped back onto that at the outer boundary based on Liouville's theorem. Then, the intensity distribution at the outer boundary is modeled by a superposition of particle flows and interpreted in physical terms. Using the cosmic-ray anisotropy observed by the Tibet AS $\gamma$ experiment [1], the latest work [8] indicated that, in the interstellar medium outside the heliosphere, the flow of cosmic rays along the interstellar magnetic field $\left(B_{\mathrm{ISM}}\right)$ is dominant, and that there is a density gradient of cosmic rays in the direction of Vela, the nearest known supernova remnant at a distance of 815 light years from the Earth. To establish a conclusive modeling, however, there are at least two technical hurdles to be cleared. Firstly, the previous work [8] employed only monochromatic energy $(4 \mathrm{TeV})$ protons in the calculation of cosmic-ray trajectories in the MHD model heliosphere, while cosmic rays observed at the Earth by the Tibet AS $\gamma$ experiment are composed of a variety of atomic nuclei with different energies. One needs to take into account the energy spectrum and the composition of observed cosmic rays in the intensity-mapping process. Secondly, the $\chi^{2} /$ ndf (number of degrees of freedom) value of the fitting between the experimental and model anisotropies was 4.5 in the previous work, which was not sufficiently good. One needs to improve the model of the intensity distribution at the outer boundary so that the $\chi^{2} /$ ndf value becomes approximately unity. In this paper, we try to solve these two problems and report the results.

\section{Intensity Mapping Method}

We use data taken by the Tibet AS $\gamma$ experiment from November 1999 to May 2010 to derive the skymap of the cosmic-ray intensity distribution at $\mathrm{TeV}$ energies. The pixelization of the sky is based on the HEALPix algorithm [9] with $N_{\text {side }}=16$, which divides the sky in our field of view $\left(-20^{\circ}<\right.$ decl. $\left.<80^{\circ}\right)$ in 2056 pixels, each of which has an approximate size of $3.7^{\circ} \times 3.7^{\circ}$.

Using detailed MC simulations of air-shower generation and detector response, we estimate the rigidity distribution of cosmic rays detected by the experiment and take it into account in the intensity-mapping process. We assume a model of the energy spectrum and the cosmic-ray chemical composition based on direct measurements [10], and generate air showers in the energy range from $0.3 \mathrm{TeV}$ and $10 \mathrm{PeV}$ using CORSIKA v7.4000 [11] with EPOS LHC [12] for the high-energy hadronic interaction model and FLUKA v2011.2b [13, 14] for the low-energy hadronic interaction model. The generated air showers are fed into the detector response simulation developed by GEANT v4.10.00 [15], and analyzed in the same way as in the experiment. Figure 1 shows the 
obtained rigidity distribution of cosmic rays detected by the experiment for five typical declination bands.

We carry out the calculation of cosmic-ray trajectories using the fourth-order Runge-Kutta method. The Earth is set at four positions around the Sun at a distance of $1 \mathrm{AU}$ on the ecliptic plane to smooth out possible seasonal effects. The sky in the declination range from $-20^{\circ}$ to $80^{\circ}$ is pixelized with $N_{\text {side }}=32$, and from the center of each pixel cosmic-ray particles are shot into the heliosphere, with their charges reversed and their rigidity spectra in Figure 1. The MHD model heliosphere that we use for cosmic-ray trajectory calculation in this presentation is identical to the one employed in the previous work [8]. The trajectories of particles are traced until they reach the 'outer boundary', which is defined as a surface where the deviation of the magnetic field strength (direction) from that outside the heliosphere becomes smaller than $0.1 \%\left(0.1^{\circ}\right)$. Then we set the intensity for a particle momentum direction at the outer boundary ( $\left.I_{\text {ISM }}\right)$ to be equal to that at the Earth $\left(I_{\mathrm{E}}\right)$, based on Liouville's theorem.

The experimental data has the declination bias - the average intensity in each declination band is normalized to unity, because the detection efficiency of the experiment along the declination direction cannot be calibrated absolutely. For this reason, deriving the $I_{\text {ISM }}$ distribution at the outer boundary is not straightforward. We take the following steps: 1) set up a model of the distribution of the cosmic-ray intensity at the outer boundary $\left.I_{\mathrm{ISM}}, 2\right)$ map $I_{\mathrm{ISM}}$ to that at the Earth $I_{\mathrm{E}}, 3$ ) normalize the average of $I_{\mathrm{E}}$ in each declination band to unity, and 4) calculate $\chi^{2}$ between the normalized $I_{\mathrm{E}}$ and the experimental data. Repeating 1) to 4 ), we obtain the best-fit model of $I_{\text {ISM }}$ that minimizes $\chi^{2}$. At step 1), we set up two models. One is expressed as:

$$
I_{\mathrm{ISM}}=1+A_{1 \|} \cos \left(\mu_{2}\right)+A_{2 \|} \cos ^{2}\left(\mu_{2}\right)+A_{1 \perp} \cos \left(\mu_{1}\right)+I_{\mathrm{CG}} .
$$

In Equation (A), $\mu_{2}$ is the angle between the momentum direction of particles on the outer boundary and $\vec{B}_{\mathrm{ISM}}, A_{1 \|}\left(A_{2 \|}\right)$ is the amplitude of the dipole (quadrupole) flow of cosmic rays along $\vec{B}_{\mathrm{ISM}}, A_{1 \perp}$ is the amplitude of the diamagnetic drift of cosmic rays perpendicular to $\vec{B}_{\mathrm{ISM}}$ that could arise from $\nabla n$, a possible density gradient of cosmic rays perpendicular to $\vec{B}_{\text {ISM }}, \mu_{1}$ is the angle between the momentum direction and $\vec{B}_{\mathrm{ISM}} \times \nabla n$. The last term $I_{\mathrm{CG}}$ represents the Compton-Getting anisotropy due to the heliospheric motion of velocity $23 \mathrm{~km} / \mathrm{s}$ with respect to the surrounding interstellar medium, which is a small correction with an amplitude of $0.03 \%$. Equation (A) contains four free fitting parameters: $A_{1 \|}, A_{2 \|}, A_{1 \perp}$, and $\alpha_{1}$, the right ascension of the $\nabla n$ direction. The declination $\delta_{1}$ of the $\nabla n$ direction is uniquely determined once the best-fit value of $\alpha_{1}$ is obtained. The other model is expressed in a series of spherical harmonics $Y_{l m}$ as:

$$
I_{\mathrm{ISM}}=1+\sum_{l=1}^{l_{\max }} \sum_{m=-l}^{l} f_{l m} Y_{l m}+I_{\mathrm{CG}},
$$

where $f_{l m}$ 's are free fitting parameters. 


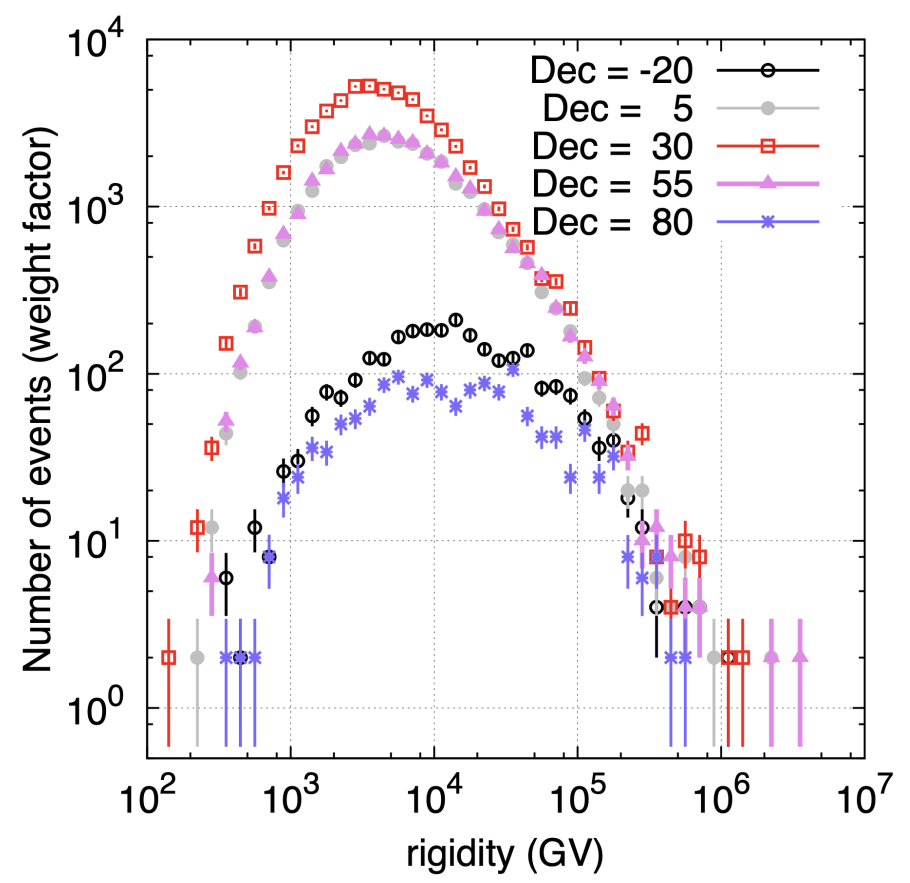

Figure 1: Rigidity distribution of cosmic rays observed by the experiment in five typical declination bands, reproduced from the detailed MC simulations.

\section{Results and Discussions}

Figure 2 shows the summary of the results. Panel (a) shows the experimental data. Panel (b) shows the best-fit model of the intensity distribution at the Earth (after the declination normalization) when we use Equation (A) for the intensity distribution at the outer boundary, and panel (c) is the best-fit model of the intensity distribution at the outer boundary. Table 1 shows the best-fit parameters obtained. The amplitude of the dipole flow $\left(A_{1 \|}\right)$ is not as dominant as in the previous work [8], and the diamagnetic drift also has a significant amplitude of $0.13 \%$, while the amplitude of quadrupole (or bi-directional) flow is less than $10 \%$ of the amplitude of the dipole flow. And the $\nabla n$ direction is $\sim 40^{\circ}$ away from that indicated in [8] which was close to the direction of Vela. The $\chi^{2} /$ ndf of this fitting is $3320 /(2056-4)=1.62$, which is significantly reduced from the previous work [8] but still needs further improvement.

Panel (d) shows the best-fit model of the intensity distribution at the Earth when we use Equation (B) with $l_{\max }=20$ for the modeling of the intensity distribution at the outer boundary, and panel (e) is the best-fit model of the intensity distribution at the outer boundary. The $\chi^{2} / \mathrm{ndf}$ of this fitting is $1658 /(2056-440)=1.03$ (p-value 22.8\%), which is acceptable. Figure 3 shows the power spectrum of the best-fit intensity distribution on the outer boundary, where

$$
C_{l}=\left(\frac{1}{4 \pi}\right)\left(\frac{1}{2 l+1}\right) \sum_{m=-l}^{l} f_{l m}^{2} .
$$

In Figure 3, we find a peculiar bump in the power spectrum around $l=7-11$. We are now investigating the cause of this bump as well as the reason why the intensity distribution at the outer 
boundary has small-scale structures with orders up to as high as $l_{\max }=20$, which seems to be unnatural. One concern is that the MHD model heliosphere used in this work is a snapshot in a positive cycle $(\mathrm{A}>0)$ of the solar magnetic dipole moment, while the duration of the experimental data corresponds to a negative cycle $(\mathrm{A}<0)$. It would be interesting to use a snapshot of the same MHD model heliosphere at a negative cycle for intensity mapping and compare the results with this work. It is noted that no significant difference between the sidereal diurnal anisotropies in $A>0$ and $\mathrm{A}<0$ epochs has been reported from the long-term observation in sub-TeV region [16].

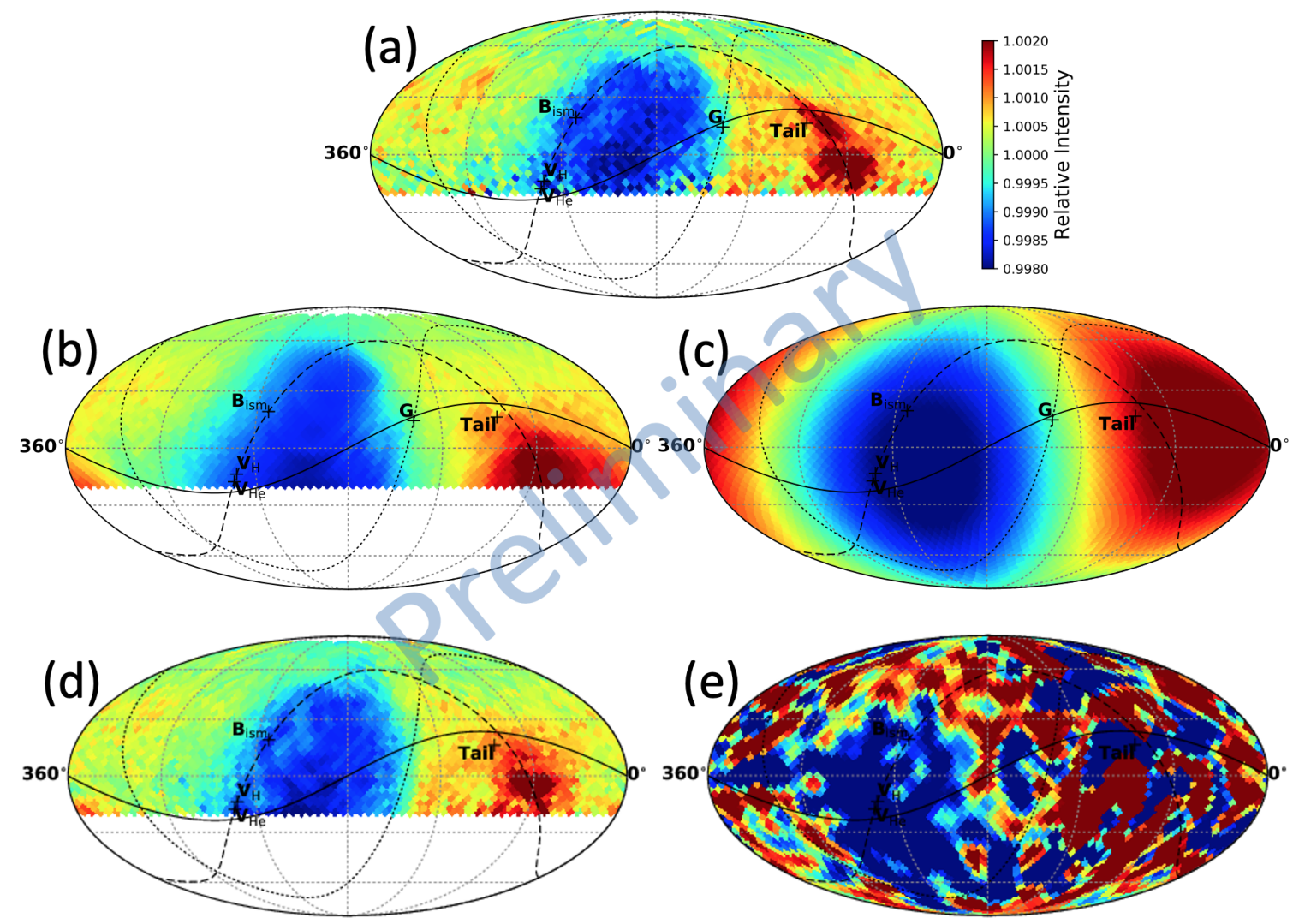

Figure 2: (a) $\mathrm{TeV}$ cosmic-ray intensity distribution observed by the Tibet AS $\gamma$ experiment from November 1999 to May 2010, (b)/(c) Best-fit model at the Earth/outer boundary using Equation (A) for the intensity distribution at the outer boundary, and (d)/(e) Best-fit model at the Earth/outer boundary using Equation (B) for the intensity distribution at the outer boundary. The color scale at the right side of (a) represents the relative intensity of cosmic rays common for all panels. The cross marks show the interstellar magnetic field orientation $\left(\mathrm{B}_{\mathrm{ISM}}\right)$ and the upstream direction of the hydrogen/helium inflows $\left(\mathrm{V}_{\mathrm{H}} / \mathrm{V}_{\mathrm{He}}\right)$ assumed in the MHD simulation of the heliosphere [8], the heliotail direction (Tail), and the best-fit direction of $\nabla n$ (G) obtained by the fitting with Equation (A). The solid, dotted and dashed lines indicate the ecliptic plane, the magnetic equator of $\mathrm{B}_{I S M}$ and the hydrogen deflection plane, respectively. 
Table 1: Best-fit parameters in the modeling with Equation (A) for the cosmic-ray intensity distribution at the outer boundary (see text).

\begin{tabular}{cccc||c}
\hline $\mathrm{A}_{1 \|}(\%)$ & $\mathrm{A}_{2 \|}(\%)$ & $\mathrm{A}_{1 \perp}(\%)$ & $\alpha_{1}\left({ }^{\circ}\right)$ & $\delta_{1}\left({ }^{\circ}\right)$ \\
$0.234 \pm 0.002$ & $0.011 \pm 0.004$ & $0.131 \pm 0.006$ & $137.5 \pm 1.4$ & $14.2 \pm 3.8$ \\
\hline
\end{tabular}

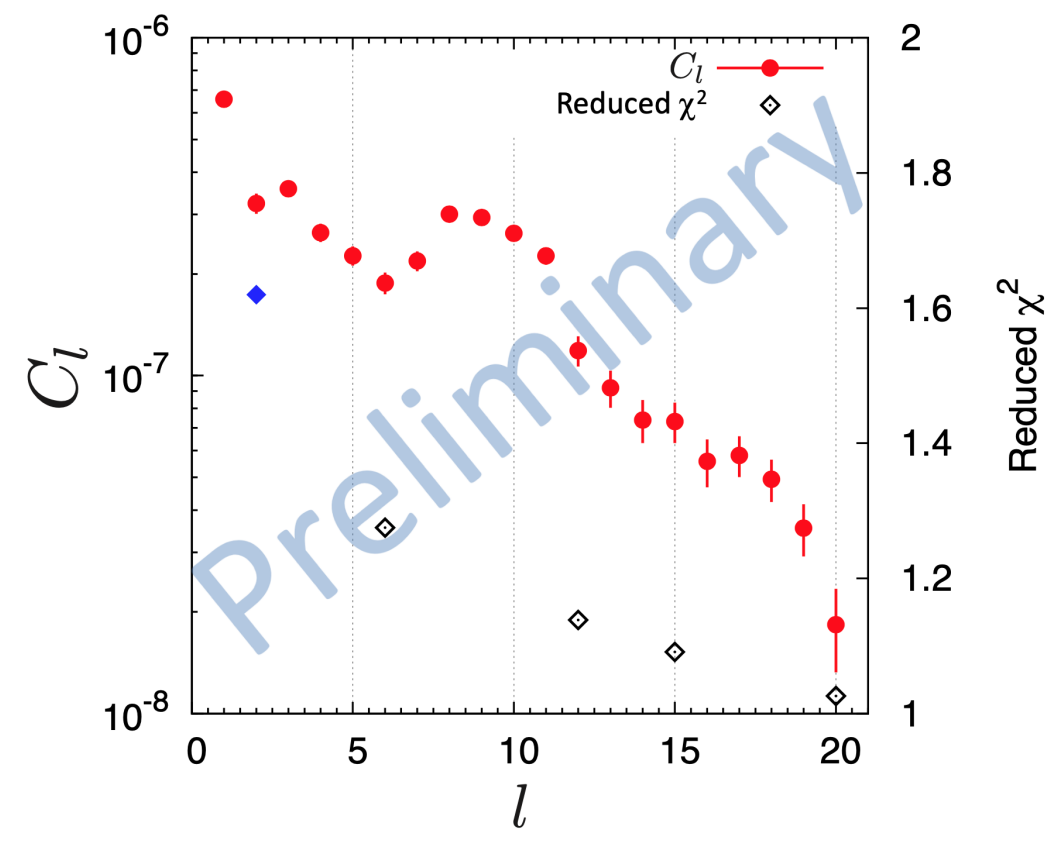

Figure 3: Power spectrum of the best-fit cosmic-ray intensity distribution on the outer boundary $\left(C_{l}\right.$ defined as Equation (C) (see text)) on the left vertical axis, along with the reduced $\chi^{2}$ on the right vertical axis in the case of the best-fit using Equation (A) (plotted at $l=2$ with a blue filled diamond) and in the case of the best-fit using Equation (B) with $l_{\max }=6,12,15$ and 20 (black open diamonds).

\section{Conclusions}

In this paper, we report the modeling of the $\mathrm{TeV}$ cosmic-ray anisotropy using the experimental data of the Tibet AS $\gamma$ experiment based on the idea of Liouville mapping. We reconstruct the rigidity distribution of cosmic rays detected by the experiment using detailed MC simulations, and take it into account when mapping the observed intensity distribution to that on the outer boundary outside the heliosphere. In addition, we improve the modeling of the cosmic-ray intensity distribution at the outer boundary to reduce the $\chi^{2}$ value of the fitting. The result shows that the cosmic-ray intensity distribution at the outer boundary has structures with angular scales as small as $\sim 10^{\circ}$ and a bump in the power spectrum around $l=7-11$, which seems to be unnatural and resulting because the MHD model heliosphere used in this work is a snapshot in a positive cycle $(A>0)$ of the solar magnetic dipole moment, while the duration of the experimental data corresponds to a negative cycle $(A<0)$. 


\section{References}

[1] M. Amenomori et al., Science, 314, 439 (2006)

[2] M. Amenomori et al., Astrophys. Space Sci. Trans., 6, 49 (2010)

[3] B. Bartoli et al., Astrophys. J., 809, 90 (2015)

[4] M. G. Aartsen et al., Astrophys. J., 826, 220 (2016)

[5] A. U. Abeysekara et al., Astrophys. J., 865, 57 (2018)

[6] A. U. Abeysekara et al., Astrophys. J., 871, 96 (2019)

[7] M. Zhang et al., Astrophys. J., 790, 5 (2014)

[8] M. Zhang et al., Astrophys. J., 889, 97 (2020)

[9] K. M. Górski et al., Astrophys. J., 622, 759 (2005)

[10] M. Shibata et al., Astrophys. J., 716, 1076 (2010)

[11] D. Heck et al., CORSIKA: a Monte Carlo Code to Simulate Extensive Air Showers Report FZKA-6019 (Forschungszentrum Karlsruhe, 1998)

[12] T. Pierog et al., Phys. Rev. C, 92, 034906 (2015)

[13] T. T. Böhlen et al., Nucl. Data Sheets, 120, 211 (2014)

[14] A. Ferrari et al., Report CERN-2005-10, INFN/TC_05/11, SLAC-R-773 (CERN European Organization for Nuclear Research, 2005)

[15] S. Agostinelli et al., Nucl. Instrum. Meth. A, 506, 250 (2003)

[16] K. Munakata et al., Astrophys. J., 712, 1100 (2010)

\section{Acknowledgements}

The collaborative experiment of the Tibet Air Shower Arrays has been conducted under the auspices of the Ministry of Science and Technology of China and the Ministry of Foreign Affairs of Japan. This work was supported in part by a Grant-in-Aid for Scientific Research on Priority Areas from the Ministry of Education, Culture, Sports, Science and Technology, and by Grants-in-Aid for Science Research from the Japan Society for the Promotion of Science in Japan. This work is supported by the National Key R\&D Program of China (No. 2016YFE0125500), the Grants from the National Natural Science Foundation of China (Nos. 11533007, 11673041, 11873065, 11773019, 11773014, 11633007, 11803011, and 11851305), and the Key Laboratory of Particle Astrophysics, Institute of High Energy Physics, CAS. This work is also supported by the joint research program of the Institute for Cosmic Ray Research (ICRR), the University of Tokyo. 


\section{Full Authors List: the Tibet AS $\gamma$ Collaboration}

M. Amenomori ${ }^{1}$, S. Asano ${ }^{2}$, Y. W. Bao ${ }^{3}$, X. J. Bi ${ }^{4}$, D. Chen ${ }^{5}$, T. L. Chen ${ }^{6}$, W. Y. Chen ${ }^{4}, \mathrm{Xu} \mathrm{Chen}^{4}$, Y. Chen ${ }^{3}, \mathrm{Cirennima}^{6}$, S. W. Cui ${ }^{7}$,

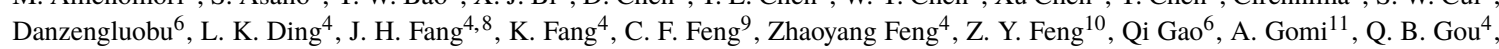
Y. Q. Guo ${ }^{4}$, Y. Y. Guo ${ }^{4}$, H. H. He ${ }^{4}$, Z. T. He ${ }^{7}$, K. Hibino ${ }^{12}$, N. Hotta ${ }^{13}$, Haibing $\mathrm{Hu}^{6}$, H. B. Hu ${ }^{4}$, K. Y. Hu ${ }^{4}, 8$, J. Huang ${ }^{4}$, H. Y. Jia ${ }^{10}$, L. Jiang ${ }^{4}$, P. Jiang ${ }^{5}$, H. B. Jin ${ }^{5}$, K. Kasahara ${ }^{14}$, Y. Katayose ${ }^{11}$, C. Kato ${ }^{2}$, S. Kato ${ }^{15}$, T. Kawashima ${ }^{15}$, K. Kawata ${ }^{15}$, M. Kozai ${ }^{16}$, D. Kurashige ${ }^{11}$, Labaciren ${ }^{6}$, G. M. Le ${ }^{17}$, A. F. Li ${ }^{18,9,4}$, H. J. Li ${ }^{6}$, W. J. Li ${ }^{4,10}$, Y. Li ${ }^{5}$, Y. H. Lin ${ }^{4,8}$, B. Liu ${ }^{19}$, C. Liu ${ }^{4}$, J. S. Liu ${ }^{4}$, L. Y. Liu ${ }^{5}$, M. Y. Liu 6 , W. Liu ${ }^{4}$, X. L. Liu Y. Nakamura ${ }^{15}$, Y. Nakazawa ${ }^{23}$, H. Nanjo ${ }^{1}$, C. C. $\mathrm{Ning}^{6}$, M. Nishizawa ${ }^{24}$, M. Ohnishi ${ }^{15}$, S. Okukawa ${ }^{11}$, S. Ozawa ${ }^{25}$, L. Qian $^{5}$, X. Qian ${ }^{5}$, X. L. Qian $^{26}$, X. B. Qu ${ }^{27}$, T. Saito ${ }^{28}$, Y. Sakakibara ${ }^{11}$, M. Sakata ${ }^{29}$, T. Sako ${ }^{15}$, T. K. Sako ${ }^{15}$, J. Shao ${ }^{4,9}$, M. Shibata ${ }^{11}$, A. Shiomi ${ }^{23}$, H. Sugimoto ${ }^{30}$, W. Takano ${ }^{12}$, M. Takita ${ }^{15}$, Y. H. Tan ${ }^{4}$, N. Tateyama ${ }^{12}$, S. Torii ${ }^{31}$, H. Tsuchiya $^{32}$, S. Udo $^{12}$, H. Wang $^{4}$, Y. P. Wang ${ }^{6}$, Wangdui ${ }^{6}$, H. R. $\mathrm{Wu}^{4}$, Q. $\mathrm{Wu}^{6}$, J. L. $\mathrm{Xu}^{5}$, L. Xue ${ }^{9}$, Z. Yang ${ }^{4}$, Y. Q. Yao ${ }^{5}$, J. Yinn ${ }^{5}$, Y. Yokoe ${ }^{15}$, N. P. Yu ${ }^{5}$, A. F. Yuan ${ }^{6}$, L. M. Zhai ${ }^{5}$, C. P. Zhang ${ }^{5}$, H. M. Zhang ${ }^{4}$, J. L. Zhang ${ }^{4}$, X. Zhang ${ }^{3}$, X. Y. Zhang ${ }^{9}$, Y. Zhang ${ }^{4}$, Yi Zhang ${ }^{33}$, Ying Zhang ${ }^{4}$, S. P. Zhao ${ }^{4}$, Zhaxisangzhu $^{6}$ and X. X. Zhou ${ }^{10}$

${ }^{1}$ Department of Physics, Hirosaki University, Hirosaki 036-8561, Japan. ${ }^{2}$ Department of Physics, Shinshu University, Matsumoto 390-8621, Japan. ${ }^{3}$ School of Astronomy and Space Science, Nanjing University, Nanjing 210093, China. ${ }^{4}$ Key Laboratory of Particle Astrophysics, Institute of High Energy Physics, Chinese Academy of Sciences, Beijing 100049, China. ${ }^{5}$ National Astronomical Observatories, Chinese Academy of Sciences, Beijing 100012, China. ${ }^{6}$ Department of Mathematics and Physics, Tibet University, Lhasa 850000, China. ${ }^{7}$ Department of Physics, Hebei Normal University, Shijiazhuang 050016, China. ${ }^{8}$ University of Chinese Academy of Sciences, Beijing 100049, China. ${ }^{9}$ Institute of Frontier and Interdisciplinary Science and Key Laboratory of Particle Physics and Particle Irradiation (MOE), Shandong University, Qingdao 266237, China. ${ }^{10}$ Institute of Modern Physics, SouthWest Jiaotong University, Chengdu 610031, China. ${ }^{11}$ Faculty of Engineering, Yokohama National University, Yokohama 240-8501, Japan. ${ }^{12}$ Faculty of Engineering, Kanagawa University, Yokohama 221-8686, Japan. ${ }^{13}$ Faculty of Education, Utsunomiya University, Utsunomiya 3218505, Japan. ${ }^{14}$ Faculty of Systems Engineering, Shibaura Institute of Technology, Omiya 330-8570, Japan. ${ }^{15}$ Institute for Cosmic Ray Research, University of Tokyo, Kashiwa 277-8582, Japan. ${ }^{16}$ Institute of Space and Astronautical Science, Japan Aerospace Exploration Agency (ISAS/JAXA), Sagamihara 252-5210, Japan. ${ }^{17}$ National Center for Space Weather, China Meteorological Administration, Beijing 100081, China. ${ }^{18}$ School of Information Science and Engineering, Shandong Agriculture University, Taian 271018, China. ${ }^{19}$ Department of Astronomy, School of Physical Sciences, University of Science and Technology of China, Hefei 230026, China. ${ }^{20}$ Department of Physics and Tsinghua Centre for Astrophysics (THCA), Tsinghua University, Beijing 100084, China. ${ }^{21}$ Tsinghua University-National Astronomical Observatories of China (NAOC) Joint Research Center for Astrophysics, Tsinghua University, Beijing 100084, China. ${ }^{22}$ Department of Astronomy, Tsinghua University, Beijing 100084, China. ${ }^{23}$ College of Industrial Technology, Nihon University, Narashino 275-8576, Japan. ${ }^{24}$ National Institute of Informatics, Tokyo 101-8430, Japan. ${ }^{25}$ National Institute of Information and Communications Technology, Tokyo 184-8795, Japan. ${ }^{26}$ Department of Mechanical and Electrical Engineering, Shangdong Management University, Jinan 250357, China. ${ }^{27}$ College of Science, China University of Petroleum, Qingdao 266555, China. ${ }^{28}$ Tokyo Metropolitan College of Industrial Technology, Tokyo 116-8523, Japan. ${ }^{29}$ Department of Physics, Konan University, Kobe 658-8501, Japan. ${ }^{30}$ Shonan Institute of Technology, Fujisawa 251-8511, Japan. ${ }^{31}$ Research Institute for Science and Engineering, Waseda University, Tokyo 162-0044, Japan. ${ }^{32}$ Japan Atomic Energy Agency, Tokai-mura 319-1195, Japan. ${ }^{33}$ Key Laboratory of Dark Matter and Space Astronomy, Purple Mountain Observatory, Chinese Academy of Sciences, Nanjing 210034, China. 